\title{
Lissencephaly type 1 due to doublecortin gene mutation
}

INSERM

\section{Source}

INSERM. (1999). Orphanet: an online rare disease and orphan drug data base.

Lissencephaly type 1 due to doublecortin gene mutation. ORPHA:2148

Type 1 lissencephaly due to doublecortin (DCX) gene mutations is a semi-dominant X-

linked disease characterised by intellectual deficiency and seizures that are more severe in male patients. 